\title{
GESTÃO DE CADEIAS DE SUPRIMENTOS VERDES: QUADRO DE TRABALHO
}

\section{GREEN SUPPLY CHAIN MANAGEMENT: A RESEARCH FRAMEWORK}

\author{
Miguel Afonso Sellitto*E-mail: sellitto@unisinos.br \\ Miriam Borchardt* E-mail: miriamb@unisinos.br \\ Giancarlo Medeiros Pereira* E-mail: gian@unisinos.br \\ Diego Augusto de Jesus Pacheco** E-mail: diegopacheco@faccat.br \\ *Universidade do Vale do Rio dos Sinos - UNISINOS, São Leopoldo, RS \\ ${ }^{* *}$ Faculdades Integrada de Taquara - FACCAT, Taquara, RS
}

\begin{abstract}
Resumo: O desenvolvimento e a estruturação do conceito de Green Supply Chain Management (GSCM) ainda não parecem estar consolidados na literatura. Igualmente, as práticas de aplicação dos conceitos da GSCM necessitam de mais investigação para que possam ser mais bem entendidas e aplicadas. O principal objetivo desse artigo é apresentar um quadro de trabalho que possa organizar e orientar a futura pesquisa em GSCM. A partir de pesquisa bibliográfica, foi possível identificar um conjunto de práticas que parecem estar maduras e analisar como alguns autores têm organizado o tema. Foram examinados estudos genéricos, que pretenderam representar a seu tempo o estado-da-arte da pesquisa, e estudos localizados, referentes a indústrias específicas ou regiões geográficas. Chegou-se a um quadro de trabalho estruturado em três grandes campos de pesquisa: estratégia, inovação, e operações. Os campos, por sua vez, se estruturaram em quatro, três e cinco áreas, respectivamente. Estratégia foi estruturada em: formulação, avaliação de desempenho, comunicação e colaboração, e barreiras e estímulos. Inovação foi estruturada em: processo, produto, e mercado. Operações foram estruturadas em: compras verdes, manufatura verde, distribuição verde, logística reversa, e gerenciamento de resíduos. Algumas pesquisas foram mencionadas como exemplos de temas pertencentes ao quadro de trabalho.
\end{abstract}

Palavras-chave: Gestão de Cadeias de Suprimentos Verdes. Gestão Ambiental. Desenvolvimento Sustentável. Logística Reversa. Ecodesign.

Abstract: The development and structuration of the concept of Green Supply Chain Management (GSCM) still does not seem to be consolidated in the literature. Also, the concept of GSCM need further research so that they can be better understood and applied. The main objective of this paper is to present a framework that can organize and guide future research in GSCM. From literature, it was possible to identify a set of practices that appear to be mature and look at how some authors have organized the subject. We examined generic studies that have attempted to represent the time the state of the art research, and localized studies relating to specific industries or geographic regions. It reached a working framework structured in three main fields: strategy, innovation, and operations. The fields are structured in four, three and five subjects, respectively. Strategy has been structured in: formulation, performance evaluation, communication and collaboration, and barriers and drives. Innovation has been structured in: process, product and market. Operations were structured in: green procurement, green manufacturing, green distribution, reverse logistics and waste management. Some studies have been cited as examples of issues belonging to the framework.

Keywords: Green Supply Chain Management. Environmental Management. Sustained Development. Reverse Logistics. Ecodesign. 


\section{INTRODUÇÃO}

A escassez dos recursos naturais e o aumento dos índices de poluição têm estimulado o debate sobre sustentabilidade e gestão ambiental. Em particular, pressões de comunidades e consumidores têm produzido regulamentações mais rigorosas sobre o tema (WALKER et al., 2008). Geralmente, os custos envolvidos em ações ambientais são considerados elevados pelas empresas e os retornos destes investimentos são calculados principalmente do ponto de vista financeiro (NOGUEIRA et al., 2011). Deste modo, cresce de importância a pressão feita por órgãos de controle e por legislações, tratados, acordos e convenções nacionais ou internacionais (DINIZ e OLIVEIRA, 2009).

Mesmo que a motivação principal das empresas ao avançar em sustentabilidade seja o atendimento a tais regulamentações, tem sido observado que práticas ambientalmente amigáveis também têm gerado aumentos de competitividade de longo prazo (PAULRAJ, 2009). Mais recentemente, empresas fabricantes de equipamentos baseados em tecnologia têm aumentado sua competitividade incorporando características ambientalmente amigáveis em seus projetos de produto (BORCHARDT et al., 2010) e em serviços associados ao produto (BORCHARDT et al., 2008).

A GSCM (ou GrSCM, Green Supply Chain Management - Gestão da Cadeia de Suprimentos Verde) visa a organizar e sistematizar práticas adotadas pelas empresas que possam promover a sustentabilidade e aumentar a competitividade na cadeia de suprimentos (SEURING e MULLER, 2008). A GSCM redesenha a cadeia de suprimentos, incorporando práticas tais como reciclagem de materiais, remanufatura, reutilização de sobras e projeto voltado ao ambiente, minimizando o impacto total da atividade industrial ao longo do ciclo de vida do produto (GREEN et al., 1998). Os autores consideram que a GSCM seja um pré-requisito para o desenvolvimento sustentável em operações industriais.

$O$ interesse acadêmico pela GSCM inclui o conceito de responsabilidade estendida de produtores, que exige que empresas focais coordenem esforços e formulem estratégias para atingir objetivos ambientais e de sustentabilidade nas cadeias de suprimentos (SHEU e TALLEY, 2011), além dos objetivos de lucratividade e atendimento a clientes (ZUCATTO et al., 2008). Tais objetivos podem exigir colaboração com fornecedores e distribuidores em ações de melhoria em processos produtivos, reprojeto de produtos com base em conceitos ambientalmente amigáveis 
(ecodesign) e redesenho de redes logísticas, incluindo rotas e fluxos reversos (MALCON, 2010).

Jabbour e Jabbour (2010) encontraram evidências de correlação positiva entre o nível de maturidade dos sistemas de gestão ambiental e o nível dos requisitos ambientais que empresas focais impõem a fornecedores. Zhu e Sarkis (2004), Rao (2002) e Rao e Holt (2005) identificaram que empresas que operam programas ambientais consistentes reduziram custos de operação da cadeia de suprimentos, enquanto que empresas com fracas ou sem iniciativas ambientais têm custos maiores para operações similares. Os autores relataram melhoria na imagem corporativa e vantagem competitiva em vendas.

Com base em tal argumentação, entende-se que a adoção da GSCM possa trazer benefícios sistêmicos e aumento de competitividade e, portanto, pode e deve ser tema de pesquisa. No entanto, os modos como objetivos ambientais podem ser atingidos ainda não parecem suficientemente consolidados e unificados na literatura (SRIVASTAVA, 2007). As principais práticas gerenciais adotadas na GSCM ainda precisam ser mais bem descritas e organizadas (TATE et al., 2010).

O objetivo geral deste artigo é apresentar um quadro de trabalho que possa classificar a pesquisa atual e direcionar a pesquisa futura. Para tanto, foi feita revisão conceitual sobre práticas adotadas em GSCM manufatureiras. Foram consultados estudos que pretenderam representar a seu tempo o estado-da-arte da pesquisa, e estudos específicos, referentes a indústrias ou regiões. Revisões de literatura podem ser úteis nos passos iniciais de uma pesquisa, principalmente quando ainda há necessidade de mais esclarecimentos e de eventuais unificações nos conteúdos a serem acomodados em um campo de conhecimento (EASTERBY-SMITH et al. 2002; MEREDITH 1993). Como o GSCM ainda apresenta lacunas conceituais importantes (SRIVASTAVA, 2007), entende-se que esforços de revisão e unificação de referências possam ser úteis neste estágio em que se encontra a pesquisa sobre o tema. $A$ principal contribuição do artigo alinha-se com objetivos de Gestão da Produção, principalmente em Logística e Gestão da Cadeia de Suprimentos e Distribuição e também com objetivos de Gestão Ambiental dos Processos Produtivos, principalmente em Produção mais Limpa e Ecoeficiência, segundo as áreas da ABEPRO. 
Após a introdução, o artigo revisa SCM (Supply Chain Management - Gestão da Cadeia de Suprimentos) e GSCM, organiza ideias extraídas de referências e apresenta e discute um quadro de trabalho para futuras pesquisas.

\section{SCM e GSCM}

Para Lambert et al. (2008), a SCM compreende os esforços de gestão e de alinhamento estratégico das ações de empresas que, juntas, atendem a um mercado. A SCM inclui planejamento, implementação e controle das operações de abastecimento e distribuição, aquisição, armazenagem e movimentação de matérias-primas, materiais intermediários e produtos acabados, desde o ponto de origem até o ponto de consumo. Também inclui eventuais retornos de materiais que podem tornar-se matérias-primas de outras cadeias. Para Cristopher (2007), a SCM constrói e gerencia uma rede de organizações, conectadas tanto a montante quanto a jusante, em processos que geram valor sob a forma de produtos ou serviços finais ao consumidor. A SCM requer que empresas parceiras de negócios compartilhem informações, riscos e benefícios e construam uma visão sistêmica e colaborativa, pois suas etapas podem ser mutuamente dependentes (MENTZER et al., 2001; LAMBERT e COOPER, 2000).

No longo prazo, a SCM preocupa-se com o formato da integração vertical, decisões sobre fazer ou comprar, infra-estrutura e tecnologia da informação. No curto prazo, trata de critérios de compra, gestão dos prazos de entrega, uso de veículos, previsões de demanda de bens e serviços e estocagem. Os principais processos de negócios são: relacionamento com clientes e serviço; gestão da demanda; atendimento de pedidos; fluxos de materiais, informações e pagamentos; compras; desenvolvimento de produtos; e retorno de bens e resíduos (COOPER et al. 1997; LAMBERT e COOPER, 2000; MENTZER et al. 2001; LARSON e HALLDORSSON, 2004; KETCHEN e HULT, 2006; KOUVELIS et al., 2006 HALLDORSSON et al. 2007).

A GSCM integra práticas ambientalmente amigáveis às práticas de gestão da cadeia (SRIVASTAVA, 2007). Os objetivos principais da GSCM são aumentar lucro e participação no mercado por meio de ações de redução de riscos e impactos ambientais e aumento da eco-eficiência ou desempenho ambiental de empresas focais e parceiros (RAO e HOLT, 2005; ZHU et al., 2005; SEURING e MULLER, 2008). O

aumento do desempenho ambiental inclui conhecer e reduzir o impacto sobre o ar, 
água, terra e formas de vida. A GSCM inclui abordagens reativas, que medem o impacto ambiental das operações e os resultados dos programas adotados; e abordagens proativas, que modificam os processos de produção para reduzir o impacto esperado sobre o ambiente (MALCON, 2010). Ambas podem ser integradas em um sistema de medição de desempenho ambiental que forneça informação necessária e suficiente para ações de controle ambiental (SELLITTO et al., 2010). Para Zucatto et al. (2008), a complexidade presente na GSCM exige uma perspectiva de análise sistêmica, multicritério e multidisciplinar, que interprete as interações. Para Mollenkopf et al. (2010), estratégias de cadeia verdes, ágeis e enxutas se relacionam mutuamente e não podem ser analisadas em separado: medidas de desempenho são afetadas pelas três, sinergicamente.

O impacto no ambiente ocorre de diversas maneiras. Sellitto et al. (2011) dividiram os impactos ambientais observados em cadeias de suprimento em: emissões atmosféricas; efluentes líquidos; geração de resíduos sólidos; e uso de recursos naturais e energéticos. Os autores mencionam que o atendimento a regulações e dispositivos legais são condições prévias, mas não necessariamente suficientes para uma operação industrial ambientalmente amigável. Os impactos devem ser observados em toda a cadeia: fornecedores, manufaturadores, montadores, distribuidores, assistentes técnicos e eventuais recicladores (ZHU e SARKIS, 2004).

Quanto às estratégias de GSCM, Wisner et al. (2005) afirmam que deve-se contemplar simultaneamente vários aspectos: normas ambientais, requisitos de clientes, envolvimento das diversas áreas da empresa, desenvolvimento de políticas ambientais específicas, comunicação com clientes e fornecedores, gestão da complexidade e controle de desempenho. Para Srivastava (2007), a implantação da GSCM exige uma estratégia para a integração do gerenciamento ambiental com as operações industriais de rotina. Para Zhu et al. (2008), a estratégia da GSCM deve envolver fornecedores, fabricantes, distribuidores, consumidores e operadores de cadeias reversas. Para Gold et al. (2008), a colaboração entre empresas é essencial para que se atinjam objetivos de desempenho econômico, ambiental e social. Recursos inter-firmas e competências emergentes da colaboração podem tornar-se fontes de vantagem competitiva, pois sua natureza complexa e causalidade ambígua podem ser difíceis de imitar por concorrentes. Routroy (2009) identifica como estimuladores da GSCM o comprometimento da alta direção, as pressões de clientes, e a ação 
reguladora e fiscalizadora de órgãos públicos. Kleindorfer et al., (2005) afirmam que empresas tendem a melhorar seu desempenho ambiental quando a pressão pública resulta em regulamentação. Às vezes, as próprias empresas pressionam por regulamentações mais severas quando acreditam que estas possam propiciar vantagem competitiva, diferenciando-as de eventuais concorrentes. Uma estratégia de GSCM pode ser verificada pela evolução do desempenho ambiental de empresas, fornecedores e clientes ( $L U$ et al., 2007). O conceito de desempenho ambiental é compreensivamente explorado em Sellitto et al. $(2010 ; 2011)$ e exemplificado em Antonov e Sellitto (2011).

Outra característica da GSCM é promover a inovação, tanto em alternativas tecnológicas de reciclagem e reuso quanto na exigência de novos materiais. Foram observados casos de ganhos expressivos de competitividade na indústria do plástico pelo uso de materiais e resinas reciclados e recuperados (FARROW et al., 2000). A inovação em GSCM materializa-se no ecodesign, que inclui o desenvolvimento de produtos mais duráveis e eficientes, livres de componentes perigosos ou de descarte problemático e facilmente desmontáveis ao fim da vida útil. A este processo chama-se recuperação de valor (LINTON et al., 2007). Exemplos de projetos baseados no Ecodesign são abundantes na literatura. Dentre muitos, Borchardt et al. (2010), McAuley (2003) e Plambeck (2007) apresentam respectivamente exemplos na indústria manufatureira, automotiva e em serviços. Nos casos estudados, os objetivos de projeto foram a redução de materiais e de energéticos ao longo da produção e do uso dos produtos projetados. Devido à complexidade presente no desenvolvimento de processos e produtos ambientalmente amigáveis, a formação de redes de cooperação e inovação pode ser importante na GSCM (MOORE e MANRING, 2009).

Autores destacaram ações operacionais importantes na GSCM. Walker et al. (2008) destacaram: uso e reuso de embalagens recicláveis, reuso de materiais, reciclagem de produtos, redução de poluentes e energéticos no transporte, remanufatura de itens defeituosos ou usados, assistência técnica para reparo de itens e recall de itens. Zhu et al. (2004) destacaram: gerenciamento interno, compras verdes, ecodesign, cooperação com o cliente, e recuperação de ativos. Dias (2006) destacou: integração das logísticas de abastecimento e distribuição com retorno e reuso de materiais; definição da reciclagem e reuso de materiais já nas fases de compras e desenvolvimento do produto. Srivastava (2007) destacou: reuso, retrabalho, reciclagem, 
remanufatura e logística reversa. Hervani et al. (2005) sintetizaram: GSCM = [Compras verdes + Manufatura verde + Distribuição verde + Logística Reversa]. Os três primeiros preocupam-se com as operações diretas. O reuso, reciclagem ou reaproveitamento de materiais é tema da logística reversa (POCHAMPALLY et al., 2009).

Apesar de observadas práticas consagradas nas empresas, tais como design de produtos sustentáveis, logística reversa e compras ambientalmente amigáveis, têm surgido evidências de que uma implementação sistêmica da GSCM não é um processo simples (NARASIMHAN e CARTER. 1998). Pesquisas referenciadas pelos autores apontaram que, algumas vezes, apesar do dano econômico do impacto ambiental, a gestão destinou recursos insuficientes para sua redução. Bangalore (2009) relatou pesquisas que apontaram projetos de melhoria ambiental sem definição clara de indicadores de desempenho e de objetivos a serem atingidos pelo programa.

\section{A PESQUISA}

O objetivo de pesquisa foi alcançado pelo método da pesquisa bibliográfica. Segundo Gil (2010), usualmente objetivos de pesquisa requerem uma revisão bibliográfica que gere um quadro de trabalho organizando a futura pesquisa e contextualizando os eventuais resultados futuros. A pesquisa bibliográfica deve considerar material publicado que foi submetido a revisão por pares, tais como: artigos em revistas científicas, teses e dissertações.

$\mathrm{Na}$ primeira parte da pesquisa, foram revisados estudos sobre GSCM. Foram incluídos estudos de revisão teórica e que propõem modos de organizar o tema e estudos que envolveram indústrias ou regiões geográficas específicas. Na segunda parte, os achados foram organizados e um quadro de referência para futuras pesquisas foi proposto. Espera-se que mais pesquisas possam refinar o quadro.

\subsection{Análise de textos em GSCM}

Iniciou-se por Srivastava (2007). A partir de extensa e compreensiva revisão de pesquisas, o autor distribuiu a abrangência da GSCM em três categorias: importância ambiental, ecodesign e operações verdes. 
A importância da GSCM está relacionada à compreensão da SCM como um objeto gerencial composto por subsistemas ambientalmente amigáveis, cujos esforços de coordenação previnam iniciativas individuais e sub-otimizações. Os benefícios podem ser intangíveis ou de mensuração direta e sua mensuração pode demandar métodos qualitativos de avaliação de desempenho. O Ecodesign envolve: (i) análise de ciclo de vida do produto, que envolve o mapeamento do fluxo de material, dos resíduos gerados na produção e no uso e na energia consumida desde a obtenção de matéria prima até a disposição final; e (ii) projeto do produto ambientalmente amigável, que envolve o uso de materiais atóxicos, de baixo impacto ambiental, fácil desmontagem, baixo consumo de energia e reaproveitáveis. As atividades operacionais envolvem manufatura verde e remanufatura, logística reversa e projeto da rede e gestão dos resíduos.

Os desdobramentos das categorias estão representados na Figura 1.

Figura 1 - Categorias e abrangência da GSCM

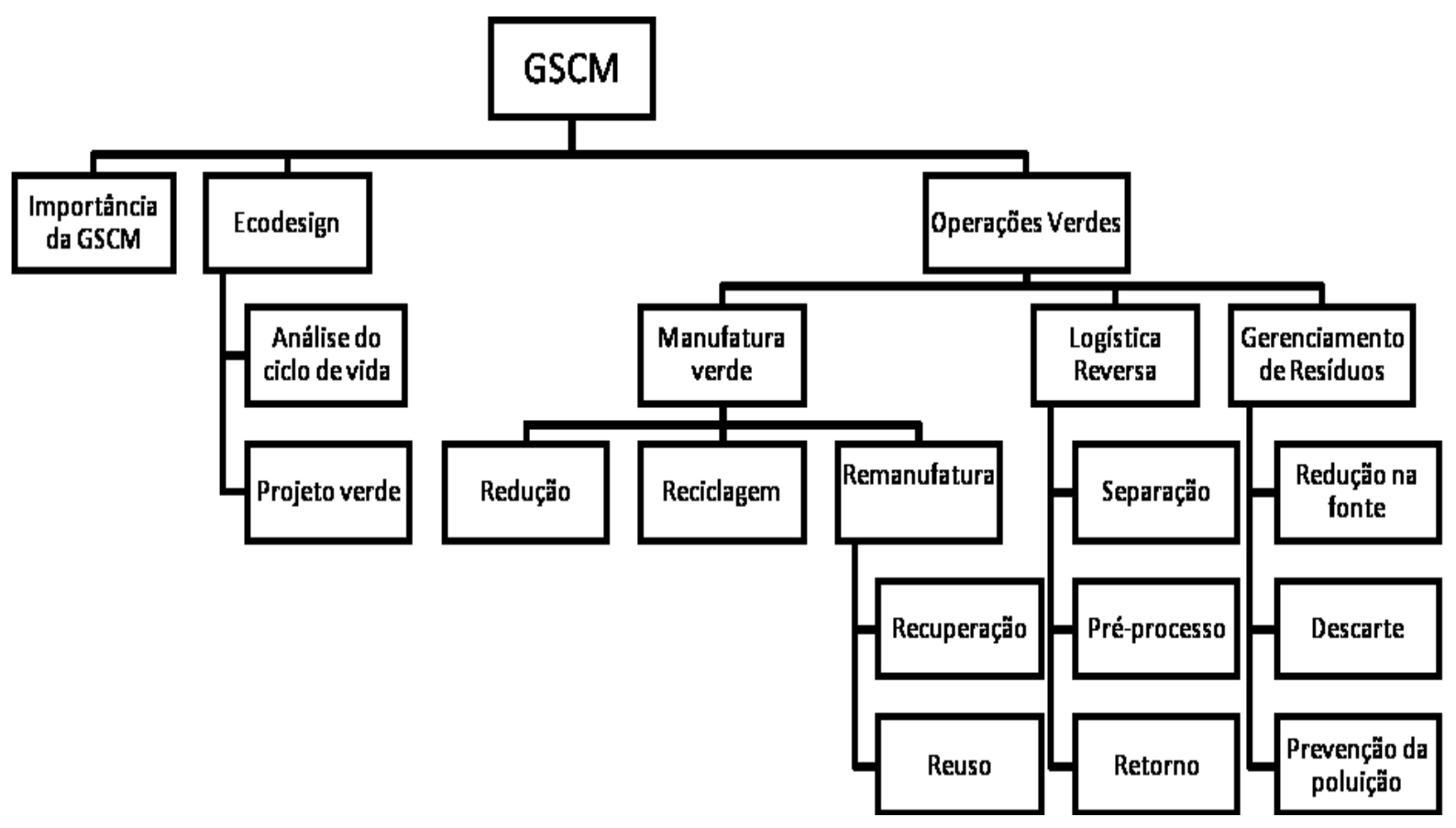

Fonte: adaptado de Srivastava (2007)

Em Seuring e Muller (2008), após revisar 191 artigos, os autores identificaram que sustentabilidade é mais do que gestão ambiental e inclui objetivos econômicos e sociais, mutuamente dependentes e em organização complexa. Segundo os autores, as empresas focais também são responsáveis pelos desempenhos ambientais e sociais de 
fornecedores e distribuidores. Os autores dividiram a GSCM em três grandes blocos: (i) pressões, barreiras e incentivos; (ii) gerenciamento verde de fornecimentos; e (iii) gerenciamento verde do produto. No primeiro bloco, incluíram o estudo das pressões legais, de consumidores, de interessados na empresa, de ONG's e de grupos de pressão, vantagens competitivas associadas à imagem e reputação da empresa e barreiras na implantação, tais como aumento de custos e complexidade na coordenação e na troca de informação, No segundo bloco, os autores incluíram o estudo de práticas de operações verdes de manufatura e transporte, atendimento a normas, medição e controle de desempenho operacional e ambiental e tecnologia de informação envolvida. No terceiro bloco, os autores incluíram como tornar o produto mais amigável ambientalmente, o que inclui o ecodesign e a análise de ciclo de vida.

Seguiu-se com Minatti et al. (2011). Os autores revisaram extensa bibliografia e separaram os métodos e técnicas encontradas em dez categorias: (i) compras verdes; (ii) fornecimentos verdes; (iii) manufatura verde; (iv) operação verde; (v) projeto verde; (vi) embalagem verde; (vii) logística reversa; (viii) sistemas de gestão ambiental (SGA); (ix) inovação verde; e $(x)$ reconhecimento do cliente. As categorias não são mutuamente excludentes. Algumas categorias apresentam sombreamentos. Compras verdes tratam da imposição de exigências ambientais por parte de empresas focais e métodos de colaboração com fornecedores. Fornecimentos verdes tratam de iniciativas ambientais dos membros da cadeia. Manufatura verde trata de procedimentos de fabricação que minimizem o impacto ambiental. Operações verdes incluem atividades não de fabricação, tais como transporte, armazenagem, inspeção, manutenção e assistência técnica. Projeto verde inclui as técnicas de ecodesign. Embalagem verde inclui reprojeto, reutilização, reaproveitamento e minimização da quantidade e material das embalagens. SGA enfocam o uso de certificações correspondentes, tais como normas das famílias ISO-14000 e OSHAS-18000 e outras. Inovação verde inclui o uso de técnicas de estímulo à criatividade e uso de novas tecnologias para redução do impacto ambiental. Reconhecimento do cliente inclui procedimentos que explicitam as expectativas do cliente em relação aos produtos, tais como rotulagens ambientais e ações de divulgação de produtos ambientalmente amigáveis.

Seguiu-se com Kleindorfer et al. (2005). Os autores revisaram a presença do tema Gestão de Operações Sustentáveis nas primeiras cinqüenta edições da revista POM. Os autores identificaram três categorias: (i) produtos verdes e desenvolvimento 
de processo; (ii) gestão de operações verdes e enxutas; e (iii) remanufatura e cadeias de suprimento em circuito fechado. Na primeira categoria, localizaram estudos sobre projetos de produtos sustentáveis e seus impactos nos resultados da cadeia. $\mathrm{Na}$ segunda, estudos que exploram sinergias entre operações verdes e operações enxutas, atendimento a regulações, responsabilidades e saúde ocupacional da mão-de-obra. $\mathrm{Na}$ terceira, retornos, desmontagens, reaproveitamento, remanufatura, recompra e revenda de itens após a entrega, em uma lógica de circuito fechado na cadeia.

Segue-se com Sarkis (2003). O autor divide o cenário da GSCM em quatro grupos de atividades: (i) ciclo operacional, composto de fornecimento, produção, distribuição, logística reversa, e embalagem; (ii) ciclo de vida do produto, composto de introdução, crescimento, maturidade; e declínio; (iii) práticas ambientais, formadas por redução, reciclagem, remanufatura, reuso e disposição de materiais; e (iv) critérios de competição: tempo, qualidade, custo e flexibilidade. Os três primeiros são antecedentes e o quarto inclui medidas de desempenho. O autor reconhece que os elementos organizados são interdependentes e recomenda o uso de métodos multicriteriais realimentados para avaliação de alternativas para a estratégia de cadeia sobre escolha dos parceiros, escolha da tecnologia, e escolha do tipo de tipo de colaboração.

Segue-se com estudos setoriais ou delimitados regionalmente. Inicia-se com Nunes e Bennett (2010), que investigaram práticas sustentáveis na indústria automotiva.

O estudo valeu-se de dados da Toyota (Japão), GM (USA) e Volkswagem (Europa). A Toyota emitiu um manual verde para seus fornecedores (Greener Supplier Guidelines - Green Purchasing Guidelines), que inclui análise do ciclo de vida dos veículos, desde o projeto do produto até a destinação final, como matéria-prima da indústria siderúrgica ou desmontagem em revendedores para uso de peças de baixa degradação, tais como airbags. A GM também tem adotado práticas de gestão do ciclo de vida de itens. Sua meta é ter em 2015 uma proporção de materiais em final de ciclo de vida, prontos para serem reusados ou recuperados, de cerca de $95 \%$ do peso do veículo. A empresa tem exigido a certificação ISO 14000 para todos os fornecedores. A Volkswagem possui sistemas avançados de reciclagem e remanufatura. A empresa também quer chegar próximo a $95 \%$ de reciclagem de materiais em fim de ciclo de vida. De forma geral, as ações de GSCM das três empresas incluem a venda de carros flex, seleção de fornecedores, transferência de tecnologias sustentáveis e sistemas logísticos mais eco-eficientes, com redução de uso de embalagens, desenvolvimento de 
embalagens retornáveis, uso de contenedores de metal em vez de papelão ou pallets de madeira, otimização de rotas de entrega e coletas de cargas e uso de combustíveis alternativos. As práticas em GSCM têm envolvido fornecedores e distribuidores. Têm se destacado a incorporação de critérios ambientais nas decisões de compra e nas relações com fornecedores, o compartilhamento de riscos ambientais e a transferência de tecnologia. Como resultado colateral, reduções de custos e desperdícios têm sido observados nas empresas, aproximando a GSCM da abordagem enxuta de gestão. O estudo apresenta extensa lista comparativa de iniciativas das três montadoras em atividades relacionadas a ecodesign, estratégia, logística e manufatura verde.

O estudo de Hsu e Hu (2008), realizado na manufatura de eletrônicos, evidenciou práticas que sustentam a GSCM na indústria. Algumas práticas relacionamse com atividades logísticas: compras verdes, auditorias ambientais para fornecedores, desenvolvimento de produtos em projetos colaborativos com fornecedores, parcerias com organizações locais de reciclagem e colaboração com indústrias do setor sobre produtos recicláveis, manuais de desmontagem de produtos, disposição final de produtos e retorno após o fim da vida útil. Algumas práticas relatadas relacionam-se a fatores humanos e de estratégia: educação e treinamento ambiental, suporte da alta gerência, integração entre setores, envolvimento da força de trabalho em causas ambientais e sociais, decisões de longo prazo com formação de parcerias duradouras, escolha de fornecedores, processos, produtos verdes. Dentre as práticas de coordenação dos esforços de cadeia, o estudo evidenciou: comunicação online dentro da companhia e com fornecedores, sistema de gerenciamento de riscos ambientais, seleção e avaliação de fornecedores e portfólio de produtos sustentáveis.

O estudo de Holt e Ghobadian (2009) pesquisou uma amostra de 149 empresas do Reino Unido e concluiu que a maioria das práticas observadas de GSCM focava principalmente em redução de custos nas atividades internas. Menos esforços nos processos de abastecimento e distribuição foram observados. Como forma de ampliar a GSCM, o estudo sugeriu o aumento da comunicação entre empresa focal, fornecedores e distribuidores e compras verdes de materiais. O estudo também evidenciou que foco excessivo em melhorias nas operações internas tem levado empresas a negligenciar uma relação proativa com a estratégia de cadeia. Por exemplo, o estudo relatou mais atividades de auditorias nos fornecedores do que de coordenação na cadeia. 
O estudo de Zhu et al. (2005) investigou práticas verdes em manufatura na China. Apoiados em revisão, os autores construíram um questionário organizado em três blocos: barreiras e estímulos a atividades verdes; práticas operacionais na cadeia; e reflexos no desempenho. O número de respostas válidas foi de 314 . O primeiro bloco apontou que existem diversos tipos e fontes de pressões, tais como: regulamentações regionais, pressões de clientes, pressões de custo e pressões e ofertas tecnológicas de empresas parceiras da cadeia. O segundo bloco apontou diversos tipos de práticas internas, tais como: sistemas internos de gestão, ecodesign, recuperação de máquinas e materiais usados e práticas combinadas e coordenadas entre empresas. O terceiro bloco apontou que práticas verdes, em algum grau e com algumas limitações, aumentaram os desempenhos ambiental, operacional e econômico das empresas.

O estudo publicado em Zhu et al. (2007; 2008) testou um modelo de mensuração de práticas de GSCM. O modelo estruturou as práticas em cinco blocos: gerenciamento ambiental interno; compras verdes; cooperação com clientes; ecodesign; e retornos financeiros com sobras e reaproveitamentos. Todos os blocos apresentaram correlação significativa com a GSCM, se bem que retorno financeiro apresentasse correlação bem mais baixa do que os demais.

O estudo de Rao e Holt (2005) organizou as práticas observadas em GSCM em construtos: compras verdes; manufatura verde; distribuição verde; competitividade sistêmica; e desempenho econômico. Os três primeiros são grandezas de entrada no modelo. $\mathrm{O}$ quarto inclui grandezas intermediárias e o quinto grandezas finais. Os autores concluíram que ações articuladas nos três primeiros construtos resultam em competitividade aumentada e conseqüentemente maior desempenho econômico.

Por fim, o estudo de Chiou et al. (2011) lançou luz na relação existente entre inovação na GSCM e aumento de desempenho ambiental e vantagem competitiva. Com base em survey incluindo 124 respondentes de oito indústrias de Taiwan, os autores concluíram que a maior influência no desempenho ambiental veio de compras verdes, ou seja, a inclusão de cláusulas ambientalmente amigáveis a contratos de fornecimento. 


\section{DISCUSSÃO}

Com base nos estudos revisados, propõe-se um quadro de trabalho para organizar e classificar a futura pesquisa em GSCM. O quadro proposto reorganizou algumas das ideias dos autores revisados, combinadas com novas perspectivas para a GSCM. O tema foi organizado em três grandes campos para futura pesquisa: estratégia; inovação; e operação.

O valor metodológico do quadro de trabalho proposto está em que ele pode ajudar a organizar a futura pesquisa em GSCM. Para um grupo que se proponha a pesquisar sobre GSCM, o quadro pode ajudar a distribuir os esforços de pesquisa. Uma produção que se distribua de modo equilibrado entre as áreas apontadas poderá ser mais contributiva, pois possivelmente não deixará de fora aspectos importantes e não se concentrará demais em poucos aspectos. Por se tratar de objeto complexo, uma estrutura pode ajudar a visualizar o todo em GSCM.

O campo da estratégia em GSCM foi estruturado em quatro áreas; (i) formulação de estratégia verde; (ii) medição e controle de estratégia verde; (iii) cooperação, adaptação, complexidade e comunicação entre partes; e (iv) barreiras e estímulos à GSCM. Procurou-se seguir um fluxo racional: inicia-se pela formulação da estratégia, segue-se pelo modelo de avaliação de desempenho, examinam-se as parcerias e os modos de coordenação que poderão ser usados e estudam-se as barreiras e os estímulos que surgem durante a execução estratégica.

Formulação de estratégia verde inclui pesquisas sobre como as cadeias podem fazer para formular objetivos e planos, alocar recursos na execução das atividades e agregar valor a clientes. Também inclui o uso de Sistemas de Gestão Ambiental (SGA). Medição e controle de estratégia verde inclui pesquisas sobre como as cadeias podem construir modelos quali-quantitativos para medição ou avaliação de desempenho ambiental e como usar estes modelos para realimentar a execução da estratégia formulada. Cooperação, adaptação, complexidade e comunicação entre partes inclui pesquisas sobre como promover a cooperação entre os membros, entender a complexidade presente na cadeia e que instrumentos tecnológicos podem ser usados para comunicação, coordenação e eventuais ações de correção nas atividades cooperadas. A área inclui pesquisas envolvendo a aplicação da teoria dos sistemas complexos adaptativos à GSCM. Por fim, barreiras e estímulos à GSCM incluem 
pesquisas sobre regulamentações, pressões de grupos e consumidores, incentivos legais, relacionamento com clientes, retornos financeiros, custos e outros aspectos que possam servir como estímulo ou barreira à implantação de cadeias verdes.

Inovação foi estruturada em três áreas: processo, projeto e mercado.

Processo inclui a adoção de técnicas inovadoras e desenvolvimento de tecnologia para reduzir consumo energético e de materiais, gerar e utilizar energias alternativas e aumentar sensivelmente a eficiência global de processos produtivos, alterando pouco os produtos. Esta área inclui desenvolvimentos físico-químicos e metalúrgicos com materiais e energéticos, experimentos de campo, métodos de Produção Mais Limpa e eventualmente reconstrução de plantas industriais. Projeto inclui a análise de produtos existentes com vistas à redução de impactos ambientais no atual processo de produção, com pequenas e puntuais alterações em processos produtivos. Em processo, altera-se mais o processo produtivo do que o produto. Em produto, altera-se mais o produto do que o processo. Ambas as áreas podem lançar mão de ferramentas clássicas do ecodesign, tais como desenvolvimento de criatividade e análise do ciclo de vida de produtos, processos e de inteiras cadeias. Inovações ambientais em mercado dizem respeito a criar novos nichos de mercado, até agora não existentes, para consumidores específicos de produtos verdes. Este consumidor não se interessaria em adquirir o produto, se este não fosse ambientalmente amigável.

Operação foi estruturada em cinco áreas: (i) compras e abastecimento verdes; (ii) manufatura verde; (iii) distribuição verde; (iv) logística reversa; e (v) destinação final e tratamento de poluentes.

As três primeiras áreas tratam de métodos gerenciais quali-quantitativos para tornar ambientalmente mais amigáveis as operações de abastecimento, manufatura e distribuição, sem mudar sensivelmente processos ou produtos. Métodos de pesquisa operacional podem ser usados para escolha de fornecedores e de procedimentos para que se atinjam objetivos verdes. Logística reversa inclui pesquisas sobre integração entre facilidades de cadeias diretas e reversas, reaproveitamento de embalagens e resíduos de uma cadeia na mesma cadeia ou em outra, remanufatura de itens para reaproveitamento e os demais aspectos tratados na bibliografia sobre o tema. Por fim, destinação final e tratamento de poluentes inclui procedimentos gerenciais e tecnológicos para disposição final e eventual tratamento físico-químico ou biológico de resíduos sólidos, efluentes líquidos ou emissões atmosféricas originadas de atividade 
industrial. Esta área inclui e integra métodos e técnicas das ciências físicas e biológicas com métodos de gerenciamento de operações.

A Figura 2 sintetiza e enumera o quadro de trabalho.

O Quadro 1 sintetiza o conteúdo de cada área do quadro de trabalho.

Figura 2 - Quadro de referência para classificação de pesquisas em GSCM

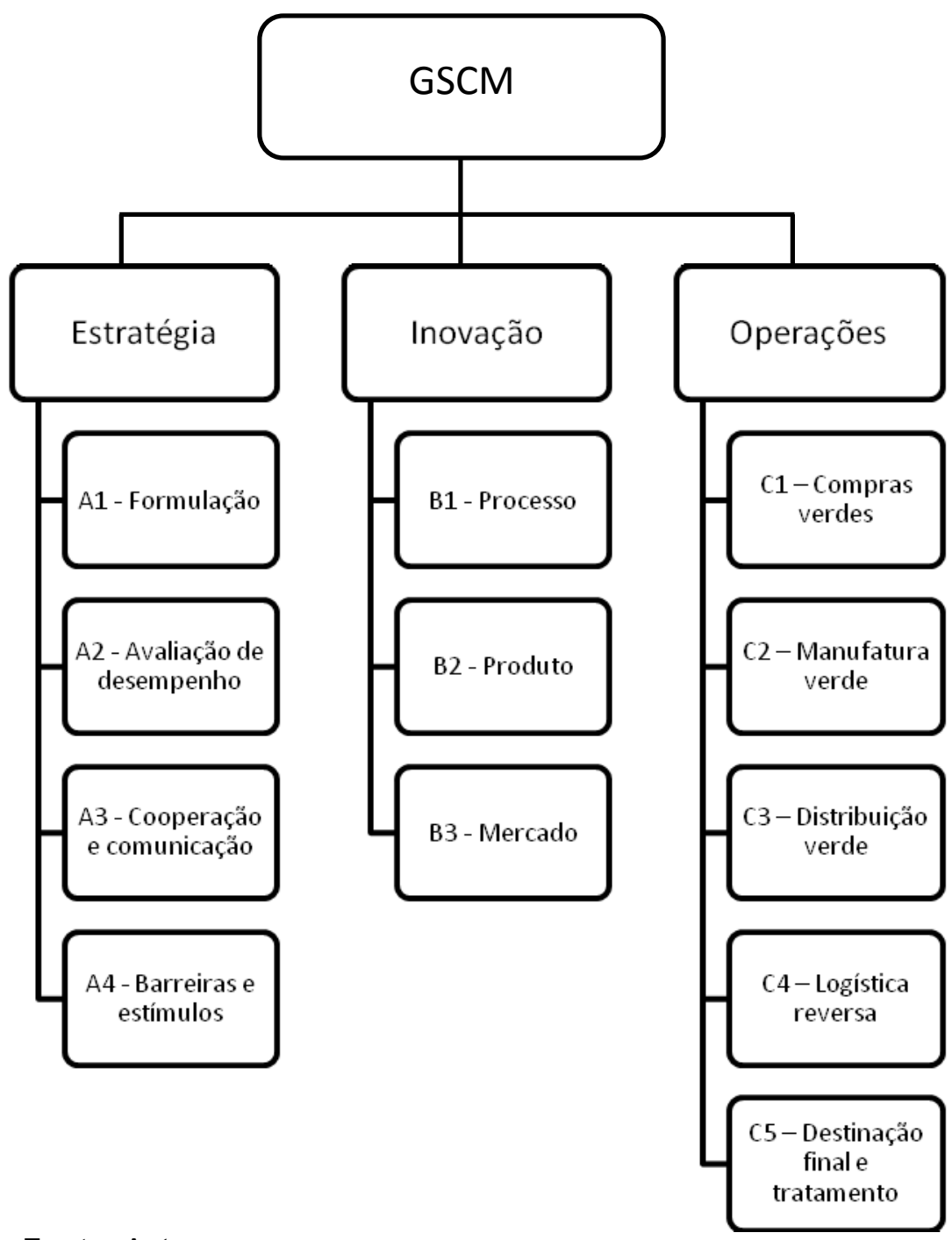

Fonte: Autores 
Quadro 1 - Síntese de conteúdos encontrados em cada área do quadro de trabalho

\begin{tabular}{|c|c|c|}
\hline $\begin{array}{l}\text { Campo de } \\
\text { estudo }\end{array}$ & Área & Conteúdos \\
\hline \multirow[t]{4}{*}{$\begin{array}{l}\text { Estratégia } \\
\text { de GSCM }\end{array}$} & Formulação - A1 & $\begin{array}{l}\text { Métodos e técnicas para formulação de objetivos e } \\
\text { planos ambientais }\end{array}$ \\
\hline & $\begin{array}{l}\text { Avaliação de desempenho - } \\
\text { A2 }\end{array}$ & $\begin{array}{l}\text { Métodos quali-quantitativos para mensuração de } \\
\text { desempenho ambiental }\end{array}$ \\
\hline & $\begin{array}{l}\text { Cooperação e comunicação- } \\
\text { A3 }\end{array}$ & $\begin{array}{l}\text { Tipologia e métodos para identificar e promover a } \\
\text { comunicação e a cooperação na GSCM }\end{array}$ \\
\hline & Barreiras e estímulos - A4 & $\begin{array}{l}\text { Fatores que podem bloquear ou acelerar a } \\
\text { implantação de iniciativas ambientalmente amigáveis } \\
\text { nas empresas }\end{array}$ \\
\hline \multirow[t]{3}{*}{$\begin{array}{l}\text { Inovação em } \\
\text { GSCM }\end{array}$} & Processo - B1 & $\begin{array}{l}\text { Inovações nos processos de produção: novas } \\
\text { tecnologias, novos materiais, novos métodos }\end{array}$ \\
\hline & Produto - B2 & $\begin{array}{l}\text { Inovações no desenvolvimento de produto: novos } \\
\text { produtos, novos métodos, análise do ciclo de vida }\end{array}$ \\
\hline & Mercado - B3 & $\begin{array}{l}\text { Criação de novos mercados: nichos de clientes que } \\
\text { não existem sem práticas ambientalmente amigáveis }\end{array}$ \\
\hline \multirow[t]{5}{*}{$\begin{array}{l}\text { Operações } \\
\text { em GSCM }\end{array}$} & Compras verdes - C1 & $\begin{array}{l}\text { Imposição e controle de requisitos ambientalmente } \\
\text { amigáveis a fornecedores }\end{array}$ \\
\hline & Manufatura verde - C2 & $\begin{array}{l}\text { Práticas de manufatura que reduzam o impacto } \\
\text { ambiental na cadeia }\end{array}$ \\
\hline & Distribuição verde - C3 & $\begin{array}{l}\text { Práticas de distribuição que reduzam o impacto } \\
\text { ambiental na cadeia }\end{array}$ \\
\hline & Logística reversa - C4 & $\begin{array}{l}\text { Ciclo fechado e recuperação de valor: retorno de } \\
\text { materiais ou resíduos para a mesma cadeia ou para } \\
\text { outras cadeias como matéria-prima ou combustível }\end{array}$ \\
\hline & $\begin{array}{l}\text { Gerenciamento de resíduos } \\
- \text { C5 }\end{array}$ & $\begin{array}{l}\text { Construção e gerenciamento de instalações de } \\
\text { reciclagem ou destinação final de resíduos sólidos. }\end{array}$ \\
\hline
\end{tabular}

Fonte: Autores

O quadro proposto relaciona-se com a bibliografia revisada.

Os três campos usados no quadro (Estratégia, Inovação, Operações) também surgem em Srivastava (2007), sob os títulos: Importância da GSCM, Ecodesign e Operações Verdes. O autor, porém parece ter dado demasiada importância às operações industriais, em comparação com os demais campos. O primeiro campo de Srivastava (2007) parece não captar tudo o que já foi proposto em estratégia verde em cadeia. Seuring e Muller (2008) também classificam as pesquisas em três campos, mas o primeiro limita-se a pressões, barreiras e incentivos para ações de sustentabilidade e o terceiro à gestão de fornecimentos. Kleindorfer et al. (2005) também usam três campos, um para gerenciamento de operações, outro para novos produtos, mas o terceiro não parece incluir todos os aspectos de interesse em estratégia. 
No campo da estratégia ambiental, estudos sobre sua formulação surgem em Rao e Holt (2005) e Minatti et al. (2009), sobre controle de desempenho em Antonov e Sellitto (2011) e Sellitto et al. (2010a; 2011), sobre coordenação e comunicação em Seuring e Muller (2008) e Srivastava (2007) e sobre barreiras e estímulos em Seuring e Muller (2008), Holt e Ghobadian (2009) e Zhu et al. (2005). No campo da inovação ambiental, estudos sobre melhorias em processos surgem em Chiou et al. (2011), em produtos em Srivastava (2007) e Kleindorfer et al. (2005) e sobre inovação em mercado em Chapple et al, 2011, Kammerer (2009) e Pajuri (2006). No campo de operações ambientais, usou-se a lógica de Hervani et al. (2005), que agrega compras, manufatura e distribuição verde e logística reversa. Acrescentou-se gerenciamento e tratamento de resíduos, também citados em Sarkis (2003). As referências citadas não são exclusivas: alguns dos campos e áreas do quadro de trabalho proposto aparecem em várias das referências revisadas.

Algumas pesquisas locais foram classificadas e podem exemplificar alguns dos itens do quadro. Vial (2010) apresentou uma estratégia de encurtamento de cadeias agroalimentares italianas que resultou em expressivos ganhos ambientais (área $\mathrm{A} 1$ ). Antonov e Sellitto (2011) e Sellitto et al. (2011; 2010a) propuseram modelos para a avaliação quali-quantitativa de desempenho ambiental em operações industriais, usando métodos multicritério de apoio à decisão (A2). Sellitto et al. (2010b) e Sellitto e Guimarães (2010) investigaram a complexidade e a comunicação presente em cadeias de suprimentos das indústrias calçadista e metal-mecânica (A3). Borchardt et al. (2009) investigaram motivações estímulos e barreiras ao ecodesign na indústria automotiva (A4). Borchardt et al. (2011) relataram ganhos econômicos e ambientais em um caso de mudança de processo de fabricação em calçado esportivo, baseada em ecodesign. A mudança implicou alterações no processo produtivo e na cadeia de abastecimento (B1).

Fleck Neto (2010) relatou os ganhos ambientais originados do reprojeto de um contenedor para transporte e movimentação de materiais industriais, tornando-o ajustável ao tamanho da carga e facilmente retornável. O contenedor vazio pode ser desmontado para retorno, ocupando $10 \%$ do volume normal. Houve mudança nas funções do produto e surgiram novos nichos de mercado, pois empresas que não usavam embalagens retornáveis passaram a usar (B2 e B3). Porn (2009) estudou práticas e propôs alternativas de gestão que tornaram mais verdes as operações de manufatura e montagem em uma empresa da indústria moveleira (área C3). Silva 
(2011) investigou métodos e técnicas de gestão que tornaram mais verde a função distribuição na indústria eletrônica (área C3). Adlmeier e Sellitto (2007) relataram os resultados de um caso envolvendo a reutilização da embalagem de cabeçotes de motores diesel exportados para os EUA (área C4). Finalmente, Gomes e Caetano (2010) investigaram como aumentar a eficiência no uso de aterros sanitários municipais compactando a carga sólida (área C5).

Espera-se que a futura pesquisa do grupo de pesquisa se distribua equilibradamente pelas áreas do quadro.

\section{CONSIDERAÇÕES FINAIS}

O objetivo deste artigo foi propor um quadro de trabalho que poderá orientar e organizar a futura pesquisa em GSCM. O método de pesquisa foi a revisão bibliográfica. A contribuição do artigo alinha-se com os temas Gestão da Produção e Gestão Ambiental dos Processos Produtivos.

Primeiramente foram revisados os conceitos de GSCM, conectando-os aos conceitos anteriores de SCM. Posteriormente, foram revisados artigos relevantes cujos objetivos foram propor organizações para o tema. Com base nas estruturas revisadas e tendo em mente as possibilidades de pesquisa futura, ao fim propôs-se um quadro de trabalho que poderá ser usado como referência para futura pesquisa. Este quadro foi baseado em alguns conceitos observados em outros estudos. Procurou-se organizar os temas de modo que resultassem linhas de pesquisa interdependentes: estratégia, inovação, operações. As linhas podem ser preenchidas por grupos de pesquisa independentes, mas também podem gerar sinergia: achados em uma linha podem eventualmente alavancar resultados em outras linhas.

O quadro de trabalho proposto poderá contribuir para organizar e priorizar os esforços de pesquisa de grupos que estejam pesquisando GSCM. Entende-se que uma pesquisa equilibrada deverá incluir de modo balanceado aspectos residentes em todas as áreas do quadro de trabalho proposto, sem concentra esforços demasiados em poucos temas e sem deixar temas sem cobertura. Espera-se que novas pesquisas possam se valer do quadro e que seus resultados possam eventualmente melhorá-lo. Esta pesquisa foi parcialmente financiada pelo CNPq. 


\section{REFERÊNCIAS}

ADLMAIER, D.; SELLITTO, M. Embalagens retornáveis para transporte de bens manufaturados: um estudo de caso em logística reversa. Produção, v.17, n.3, p.395-406, 2007.

ANTONOV, P.; SELLITTO, M. Avaliação de desempenho ambiental: estudo de caso na indústria papeleira. Produção Online, v.11, n.4, p.1059-1085, 2011.

BORCHARDT, M.; POLTOSI, L.; SELLITTO, M.; PEREIRA, G. Adopting ecodesign practices: Case study of a midsized automotive supplier. Environmental Quality Management, v.19, n.1, p.7-22, 2009.

BORCHARDT, M.; SELLITTO, M.; PEREIRA, G. Sistemas produto-serviço: referencial teórico e direções para futuras pesquisas. Produção Online, v.10, n.4, p.818-836, 2010.

BORCHARDT, M.; SELLITTO, M.; PEREIRA, G.. Serviços de pós-venda para produtos fabricados em base tecnológica. Produção Online, v.8, n.1, p.1-25, 2008.

BORCHARDT, M.; WENDT, M.; PEREIRA, G.; SELLITTO, M. Redesign of a component based on ecodesign practices: environmental impact and cost reduction achievements. Journal of Cleaner Production, v.19, n.1, p.49-57, 2010.

CHAPPLE, K.; KROLL, C.; LESTER, T.; MONTERO, S. Innovation in the green economy: An extension of the regional innovation system model? Economic Development Quarterly, v.25, n.1, p.5-25, 2011.

CHIOU, T.; CHAN, K.; LETTICE, F.; CHUNG, S. The Influence of Greening the Suppliers and Green Innovation on Environmental Performance and Competitive Advantage in Taiwan. Transportation Research Part E, v.47, n.6, p.822-836, 2011.

CHRISTOPHER, M. Logística e gerenciamento da cadeia de suprimentos. S. Paulo: Thomson, 2007.

COOPER, M.; LAMBERT, D.; PAGH, J. Supply chain management: more than a new name for logistics. The International Journal of Logistics Management, v.8, n.1, p.1-14, 1997.

DIAS, S. Há vida após a morte: um (re)pensar estratégico para o fim da vida das embalagens. Gestão \& Produção, v.13, n.3, p.463-474, 2006.

DINIZ, A.; OLIVEIRA, I. Influência das fontes de alumínio secundário na geração de escória: uma análise estatística. Produção Online, v. 9, n. 2, p. 284-302, 2009.

EASTERBY-SMITH, M., THORPE, R., LOWE, A. Management research: An Introduction. Londres: Sage Publications, 2002 
FARROW, P.; JOHNSON, R.; LARSON, A. Entrepreneurship, innovation, and sustainability strategies at walden paddler. Interfaces, v.30, n.3, p.215-225, 2000.

FLECK NETO, P. Análise da contribuição para a competitividade empresarial de um contenedor retornável sob a perspectiva da logística reversa. Dissertação (Mestrado) - Programa de Pós-graduação em Engenharia de Produção e Sistemas. Unisinos. São Leopoldo, 2010.

FLEISCHMANN, M.; KRIKKE, H.; DEKKER, R.; FLAPPER, S. A characterization of logistics networks for product recovery. Omega, v.28, n.6, p.653-66, 2000.

GIL, A. Como elaborar projetos de pesquisa. São Paulo: Atlas, 2001.

GOLD, S.; SEURING, S.; BESKE, P. Sustainable supply chain management and inter-organizational resources: a literature review. Corporate Social Responsibility and Environmental Management, v.17, n.4, p.230-245, 2009.

GOMES, L; CAETANO, M. Municipal Solid Waste Sanitary Landfill Compressibility Study with Linear Regression Application. Soils \& rocks, v.33, n.1, p.145-157, 2010.

GREEN, K.; MORTON, B.; NEW, S. Green Purchasing and Supply Policies: Do They Improve Company's Environmental Performance? Supply Chain Management: An International Journal, v. 3, n. 2, p. 89-95. 1998.

HALLDORSSON, A.; KOTZAB, H.; MIKKOLA, J.; SKJOETT-LARSEN, T. Complementary theories to supply chain management. Supply Chain Management: An International Journal, v.12, n.4, p.284-296, 2007.

HERVANI, A.; HELMS, M.; SARKIS, J. Performance measurement for green supply chain management. Benchmarking: An international Journal, v.12, n.4, p.330-353, 2005.

HOLT, D.; GHOBADIAN, A. An empirical study of green supply chain management practices amongst UK manufacturers. Journal of Manufacturing Technology Management, v.20, n.7, p.933 - 956, 2009.

HSU, C.; HU. A. Green supply chain management in the electronic industry. International Journal of Environmental Science and Technology, v.5, n.2, p.205216, 2008.

JABBOUR, A.; JABBOUR, C. Are supplier selection criteria going green? case studies of companies in Brazil. Industrial Management \& Data Systems, v.109, n.4, p.477-495, 2009.

KAMMERER, D. The effects of customer benefit and regulation on environmental product innovation. Empirical evidence from appliance manufactures in Germany. Ecological Economics, v.68, n.8-9, p. 2285 - 2295, 2009.

KETCHEN JR., G.; HULT, T. Bridging organization theory and supply chain management: The case of best value supply chains. Journal of Operations Management, v.25, n.2, p.573-580, 2006. 
KLEINDORFER, P.; SINGHAL, K.; WASSENHOVE, L. Sustainable operations management. Production and Operations Management, v.14, n.4, p.482-492, 2005.

KOUVELIS, P.; CHAMBERS, C.; WANG, H. Supply Chain Management Research and Production and Operations Management: Review, Trends, and Opportunities. Production and Operations Management, v.15, n.3, p.449-469, 2006.

LAMBERT, D. Supply Chain Management: processes, partnerships, performance. Sarasota: Supply Chain Management Institute, 2008.

LAMBERT, D., COOPER, M. Issues in supply chain management. Industrial Marketing Management, v.29, n.1, p.65-83, 2000.

LARSON, P.; HALLDORSSON, A. Logistics versus supply chain management: an international survey. International Journal of Logistics: Research \& Application, v.7, n.1, p.17-31, 2004.

LINTON, J.; KLASSEN, R.; JAYARAMAN, V. Sustainable supply chains: an introduction. Journal of Operations Management, v.25, n.6, p.1075-1082, 2007.

MALCON, J. Keeping orangutans out of the supply chain. Inside Supply Management, v.21, n.5, p.22-24, 2010.

MC AULEY, J. Global sustainability and key needs in future automotive design. Environmental Science and Technology, v.37, n.23, p.5414-5416, 2003.

MENTZER, J.; DE WITT, W.; KEEBLER, J.; MIN, S.; NIX, N.; SMITH, C.; ZACHARIA, Z. Defining supply chain management. Journal of Business Logistics, v.22, n.2, p.1-25, 2001.

MEREDITH, J. Theory building through conceptual methods. International Journal of Operations \& Production Management, v.13, n.1, p.3-11, 1993.

MEYER, H. Many happy returns. The Journal of Business Strategy, v.30, n.2, p.27-31, 1999.

MINATTI, C.; ALBERTON, A.; MARINHO, S. Direções e construtos do green supply chain management. SIMPOI, 14,. 2011. Anais... FGV, São Paulo, 2011.

MOLLENKOPF, D,; STOLZE, H.; TATE, W.; UELTSCHY, M. Green, lean, and global supply chains. International Journal of Physical Distribution \& Logistics Management, v. 40, n.1/2, p.14-41, 2010.

MOORE, S.; MANRING, S. Manufacturing strategy development in small and medium sized enterprises for sustainability and increased value creation. Journal of Cleaner Production, v.17, n.2, p.276-282, 2009.

NOGUEIRA, A.; PERES, A.; CARVALHO, E. Avaliação do risco ambiental utilizando FMEA em um laticínio na região de Lavras - MG. Produção Online, v.11, n.1, p. 194-209, 2011. 
NUNES, B.; BENNETT, D. Green operations initiatives in the automotive industry. Benchmarking: An International Journal, v.17, n.3, p.396-420, 2010.

NUNES, B.; MARQUES JR, S.; RAMOS, R. A theoretic approach for green supply chain. WORLD CONFERENCE ON PRODUCTION AND OPERATION MANAGEMENT, 2,. 2004. Anais... POMS. Cancun, 2004.

PAJURI, D. Eco-innovation and new product development: understanding the influences on market performance. Technovation, v.26, n.1, p.76-85, 2006.

PAULRAJ, A. Environmental motivations: a classification scheme and its impact on environmental strategies and practices. Business Strategy and the Environment, v.18, n.7, p.453-468, 2009.

PLAMBECK, E. The greening of Wal-Mart's supply chain. Supply Chain Management Review, v.11, n.5, p.18-25, 2007.

POCHAMPALLY, K.; GUPTA, S.; GOVINDAN, K. Metrics for performance measurement of a reverse/closed-loop supply chain. International Journal of Business Performance and Supply Chain Modelling, v.1, n.1, p. 8-32, 2009.

PORN, D. Análise das práticas do desenvolvimento de móveis estofados de alto padrão em duas empresas de pequeno porte à luz dos conceitos da ecoeficiência. Dissertação (Mestrado)- Programa de Pós-graduação em Engenharia de Produção e Sistemas. Unisinos. São Leopoldo, 2009.

RAO, P. Greening the supply chain: a new initiative in south east Asia. International Journal of Operations and Production Management, v.22, n.6, p.632 - 655, 2002.

RAO, P.; HOLT, D. Do green supply chains lead to competitiveness and economic performance? International Journal of Operations \& Production Management, v.25, n.9, p.898-916, 2005.

ROUTROY, S. Antecedents and drivers for green supply chain management implementation in manufacturing environment. The ICFAI University Journal of Supply Chain Management, v.6, n.1, p.20-35, 2009.

SARKIS, J. A strategic decision framework for green supply chain management. Journal of Cleaner Production, v.11, n.4, p.397-409, 2003.

SELLITTO, M., BORCHARDT, M.; PEREIRA, G. Modelagem para avaliação de desempenho ambiental em operações de manufatura. Gestão \& Produção, v.17, n.1, p.95-109, 2010a.

SELLITTO, M., BORCHARDT, M.; PEREIRA, G., GUIMARÃES, M. Relative complexity measurement of a supply chain based on information theory.

Proceedings of the International Multi-Conference on Complexity, Informatics and Cybernetics: IMCIC 2010. Orlando: International Institute of Informatics and Systemics, $2010 \mathrm{~b}$. 
SELLITTO, M., GUIMARÃES, M. Cálculo da complexidade organizacional em dois arranjos produtivos da indústria calçadista. Produto \& Produção, v.11, n.3, p.29-44, 2010.

SELLITTO, M.; BORCHARDT, M.; PEREIRA, G.; GOMES, L. Environmental performance assessment in transportation and warehousing operations by means of categorical indicators and multicriteria preference. Chemical Engineering Transactions, v.25, n.1, p.291-296, 2011.

SEURING, S.; MULLER, M. From a literature review to a conceptual framework for sustainable supply chain management. Journal of Cleaner Production, v.16, n.9, p.1699-1710, 2008.

SHEU, J.; TALLEY, W. Green supply chain management: trends, challenges, and solutions. Transportation Research Part E, v.47, n.6, p.791-792, 2011.

SILVA, L. Práticas e incentivos à comercialização de produtos com concepção green: um estudo de caso múltiplo na cadeia de comercialização. Dissertação (Mestrado) - Programa de Pós-graduação em Engenharia de Produção e Sistemas. Unisinos. São Leopoldo, 2011.

SRIVASTAVA, S. Green supply-chainmanagement: a state-ofthe-art literature review. International Journal of Management Reviews,v.9, n.1, p.53-80, 2007.

TATE, W.; ELLRAM, L.; KIRCHOFF, J. Corporate social responsibility reports: a thematic analysis related to supply chain management. Journal of Supply Chain Management, v.46, n.1, p.19-44, 2010.

VIAL, L. Análise de filiera corta italiana: encurtando distâncias entre produtores e consumidores. Dissertação (Mestrado) - Programa de Pós-graduação em Engenharia de Produção e Sistemas. Unisinos. São Leopoldo, 2010.

WALKER, H.; SISTOB, L.; MCBAINC, D. Drivers and barriers to environmental supply chain management practices: Lessons from the public and private sectors. Journal of Purchasing \& Supply Management, v.14, n.1, p.69-85, 2008.

WISNER, J.; LEONG, G.; TAN, K. Principles of supply chain management: a balanced approach. Mason, Ohio: Thomson South-Western, 2005.

ZHU, Q.; SARKIS, J. Relationships between operational practices and performance among early adopters of green supply chain management practices in Chinese manufacturing enterprises. Journal of Operations Management, v.22, n.3, p.265289, 2004.

ZHU, Q.; SARKIS, J.; GENG, Y. Green supply chain management in China: pressures, practices and performance. International Journal of Operations and Production Management, v.25, n.5, p.449-468, 2005.

ZHU, Q.; SARKIS, J.; LAI, K. Confirmation of a measurement model for green supply chain management practices implementation. International Journal of Production Economics, v.111, n.2, p.261-273, 2008. 
ZHU, Q.; SARKIS, J.; LAI, K. Green supply chain management: pressures, practices and performance within the chinese automobile industry. Journal of Cleaner Production, v.15, n.11/12, p.1041-1052, 2007.

ZHU, Q.; SARKIS, J.; LAI, K. Initiatives and outcomes of green supply chain management implementation by Chinese manufacturers. Journal of Environmental Management, v.85, n.2, p.179-189, 2007.

ZUCATTO, L.; VEIGA, C.; EVANGELISTA, M. Estudo comparativo entre as abordagens de Supply Chain Management e Green Supply Chain Management na perspectiva da sustentabilidade. In: ENEGEP, 28,. 2008. Anais ... Rio de Janeiro, 2008.

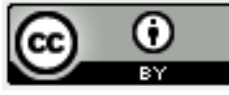

Artigo recebido em 07/12/2011 e aceito para publicação em 13/08/2012. 